\title{
Toxicokinetic studies in piglets reveal age-related differences in systemic exposure to zearalenone, zearalenone-14-glucoside and zearalenone-14-sulfate
}

Amelie Catteuw ${ }^{1}$, Mathias Devreese ${ }^{1}$, Siegrid De Baere $^{1}$, Gunther Antonissen ${ }^{1,2}$, Bart Huybrechts ${ }^{3}$, Lada Ivanova ${ }^{4}$, Silvio Uhlig ${ }^{4}$, Ann Martens ${ }^{5}$, Sarah De Saeger ${ }^{6}$, Marthe De Boevre $^{6}$, Siska Croubels ${ }^{1 *}$

${ }^{1}$ Department of Pharmacology, Toxicology and Biochemistry, Faculty of Veterinary Medicine, Ghent University, Salisburylaan 133, 9820 Merelbeke, Belgium

${ }^{2}$ Department of Pathology, Bacteriology and Avian Diseases, Faculty of Veterinary Medicine, Ghent University, Salisburylaan 133, 9820 Merelbeke, Belgium

${ }^{3}$ Sciensano, Juliette Wytsmanstraat 14, 1050 Elsene, Belgium

${ }^{4}$ Toxinology Research Group, Norwegian Veterinary Institute, Ullevålsveien 68, 0454 Oslo, Norway

${ }^{5}$ Department of Surgery and Anaesthesiology of Domestic Animals, Faculty of Veterinary Medicine, Ghent University, Salisburylaan 133, 9820 Merelbeke, Belgium

${ }^{6}$ Department of Bioanalysis, Faculty of Pharmaceutical Sciences, Ghent University, Ottergemsesteenweg 460, 9000 Ghent, Belgium

* Corresponding author: Email: siska.croubels@UGent.be 
Supplementary Table 1 Results of the linearity (r, 1/x weighted), recovery, standard error of the mean (SEM), limit of detection (LOD), limit of quantification (LOQ), signal suppression/enhancement (SSE) and total relative standard deviation $\left(\mathrm{RSD}_{\text {total }}\right)$ for the LC-HRMS validation of zearalenone $(\mathrm{ZEN}), \alpha / \beta$-zearalenol $(\alpha / \beta$ ZEL), $\alpha / \beta$-zearalanol ( $\alpha / \beta$-ZAL), ZEN-14-glucuronide (ZEN14GlcA), $\alpha$-ZEL-14-glucuronide ( $\alpha$-ZEL14GlcA), $\alpha$-ZEL-7-glucuronide ( $\alpha$-ZEL7GlcA), $\beta$-ZEL-14-glucuronide ( $\beta$-ZEL14GlcA), $\beta$-ZEL-16-glucuronide ( $\beta$ ZEL16GlcA), zearalenone-14-glucoside (ZEN14G) and zearalenone-14-sulfate (ZEN14S) in pig plasma

\begin{tabular}{lccccccc}
\hline & $\begin{array}{c}\text { Recovery } \\
\%\end{array}$ & $\begin{array}{c}\text { SEM, } \\
\%\end{array}$ & $\begin{array}{c}\text { LOD, } \\
\text { ng/mL }\end{array}$ & $\begin{array}{c}\text { LOQ, } \\
\text { ng/mL }\end{array}$ & $\begin{array}{c}\text { SSE, } \\
\%\end{array}$ & $\begin{array}{c}\text { RSD }_{\text {total }}^{2}, \\
\%\end{array}$ & $\mathrm{r}$ \\
\hline \hline$\beta$-ZEL16GlcA & 89 & 10 & 2.9 & 9.7 & 93 & 30 & 0.9953 \\
$\beta$-ZEL14GlcA & 86 & 4 & 1.5 & 5.1 & 75 & 19 & 0.9971 \\
$\alpha$-ZEL14GlcA & 85 & 4 & 1.4 & 4.6 & 94 & 21 & 0.9957 \\
$\alpha$-ZEL7GlcA & 95 & 3 & 1.4 & 4.8 & 80 & 16 & 0.9980 \\
ZEN14GlcA & 89 & 4 & 0.65 & 2.2 & 96 & 23 & 0.9961 \\
$\alpha-Z A L$ & 98 & 3 & 1.0 & 3.3 & 85 & 17 & 0.9979 \\
$\beta$-ZAL & 106 & 3 & 1.1 & 3.6 & 71 & 19 & 0.9972 \\
$\alpha-Z E L$ & 92 & 3 & 1.3 & 4.4 & 122 & 16 & 0.9961 \\
$\beta$-ZEL & 107 & 4 & 0.75 & 2.5 & 91 & 19 & 0.9976 \\
ZEN & 101 & 3 & 0.68 & 2.3 & 129 & 18 & 0.9983 \\
ZEN14G & 97 & 13 & 0.52 & 1.7 & 37 & 23 & 0.9992 \\
ZEN14S & - & - & 11 & 36 & 97 & - & 0.9995 \\
\hline
\end{tabular}

${ }^{1}$ Arithmetic mean, $\mathrm{n}=6$, spike experiments at $15 \mathrm{ng} / \mathrm{mL}$ level.

${ }^{2}$ Based on repeatability relative standard deviation (RSDr) and reproducibility relative standard deviation $\left(\mathrm{RSD}_{\mathrm{R}}\right)$ at $15 \mathrm{ng} / \mathrm{mL}$ level.

Acceptability ranges: $r \geq 0.98$; Recovery: concentration calibrator $\geq 10 \mathrm{ng} / \mathrm{mL} \rightarrow 60 \%$ to $120 \%$; RSD: $15 \mathrm{ng} / \mathrm{mL} \rightarrow \leq$ $40 \%$.

- = not available 
Supplementary Table 2 Results of the linearity (r, 1/x weighted), recovery, limit of detection (LOD), limit of quantification (LOQ) and signal suppression/enhancement (SSE) for the LC-HRMS validation of zearalenone (ZEN), $\alpha / \beta$-zearalenol ( $\alpha / \beta$-ZEL), $\alpha / \beta$-zearalanol ( $\alpha / \beta$-ZAL), ZEN-14-glucuronide (ZEN14GlcA), $\alpha$-ZEL-14glucuronide ( $\alpha$-ZEL14GlcA), $\alpha$-ZEL-7-glucuronide ( $\alpha$-ZEL7GlcA), $\beta$-ZEL-14-glucuronide ( $\beta$-ZEL14GlcA), $\beta$ ZEL-16-glucuronide ( $\beta$-ZEL16GlcA), zearalenone-14-glucoside (ZEN14G) and zearalenone-14-sulfate (ZEN14S) in pig urine

\begin{tabular}{lccccc}
\hline & Recovery $^{1}, \%$ & LOD, ng/mL & LOQ, ng/mL & SSE, \% & $\mathrm{r}$ \\
\hline \hline$\beta$-ZEL16GlcA & 67 & 0.11 & 0.38 & 153 & 0.9989 \\
$\beta$-ZEL14GlcA & 69 & 0.16 & 0.54 & 139 & 0.9993 \\
$\alpha$-ZEL14GlcA & 75 & 0.40 & 1.3 & 115 & 0.9985 \\
$\alpha$-ZEL7GlcA & 81 & 0.27 & 0.90 & 105 & 0.9988 \\
ZEN14GlcA & 76 & 1.2 & 3.9 & 125 & 0.9993 \\
$\alpha-Z A L$ & 94 & 1.2 & 4.1 & 73 & 0.9993 \\
$\beta$-ZAL & 105 & 2.1 & 6.8 & 72 & 0.9933 \\
$\alpha-Z E L$ & 116 & 0.95 & 3.2 & 74 & 0.9942 \\
$\beta$-ZEL & 11 & 1.8 & 5.9 & 84 & 0.9951 \\
ZEN & 102 & 1.1 & 3.8 & 127 & 0.9978 \\
ZEN14G & 87 & 0.51 & 1.7 & 23 & 0.9992 \\
ZEN14S & - & 29 & 97 & 147 & 0.9988 \\
\hline
\end{tabular}

${ }^{1}$ spike experiments at $15 \mathrm{ng} / \mathrm{mL}$ level, $\mathrm{n}=1$

Acceptability ranges: $r \geq 0.98$.

- = not available 
Supplementary Table 3 Comparison of the absorbed fraction (FRAC) and toxicokinetic parameters after intravenous (IV) and per oral (PO) administration of zearalenone (ZEN) and zearalenone-14-glucoside (ZEN14G) between 4- and 8-week old pigs.

\begin{tabular}{|c|c|c|c|c|}
\hline Parameter & 4-week-old & $*$ & 8-week-old & p-value \\
\hline \multicolumn{5}{|c|}{ ZEN administration (IV/PO) } \\
\hline $\operatorname{CLzen}(\mathrm{L} / \mathrm{kg} * \mathrm{~h})$ & $7.7 \pm 6.5$ & $=$ & $6.7 \pm 2.7$ & 0.854 \\
\hline Vdzen $(\mathrm{L} / \mathrm{kg})$ & $3.4 \pm 1.6$ & $<$ & $7.3 \pm 0.78$ & 0.009 \\
\hline ke $\left(h^{-1}\right)$ & $1.7 \pm 0.78$ & $=$ & $0.83 \pm 0.57$ & 0.200 \\
\hline $\mathbf{t}_{1 / 2 \mathrm{el}}(\min )$ & $35 \pm 19$ & $=$ & $57 \pm 27$ & 0.252 \\
\hline $\mathbf{C}_{0}(\mathrm{nmol} / \mathrm{mL})$ & $0.39 \pm 0.13$ & $>$ & $0.15 \pm 0.14$ & 0.036 \\
\hline FRAC (\%) & $71 \pm 29$ & $=$ & $61 \pm 20$ & 0.746 \\
\hline $\mathbf{C}_{\max }(\mathrm{nmol} / \mathrm{mL})$ & $0.11 \pm 0.053$ & $<$ & $0.35 \pm 0.16$ & 0.023 \\
\hline $\mathbf{t}_{\max }(\min )$ & $26 \pm 15$ & $=$ & $20 \pm 10$ & 0.618 \\
\hline \multicolumn{5}{|c|}{ ZEN14G administration (IV/PO) } \\
\hline CLzen14G (L/kg*h) & $5.8 \pm 2.5$ & $=$ & $3.7 \pm 1.8$ & 0.303 \\
\hline QZEN14G $(\mathrm{L} / \mathrm{kg} * \mathrm{~h})$ & $2.0 \pm 0.26$ & $>$ & $1.1 \pm 0.22$ & 0.139 \\
\hline CLzeN $(\mathrm{L} / \mathrm{kg} * \mathrm{~h})$ & $7.7 \pm 0.0$ & $=$ & $10 \pm 2.1$ & 0.001 \\
\hline $\begin{array}{l}\text { CLZEN14G } \rightarrow \text { ZEN } \\
\left(\mathrm{L} / \mathrm{kg}^{*} \mathrm{~h}\right)\end{array}$ & $3.4 \pm 1.2$ & $=$ & $3.0 \pm 0.60$ & 0.570 \\
\hline Vdzen (L/kg) & $4.9 \pm 2.0$ & $=$ & $6.7 \pm 0.0$ & 0.752 \\
\hline $\operatorname{VcZEN14G}(\mathrm{L} / \mathrm{kg})$ & $0.64 \pm 0.18$ & $=$ & $0.57 \pm 0.33$ & 0.212 \\
\hline VpZen14G $(\mathrm{L} / \mathrm{kg})$ & $0.60 \pm 0.18$ & $=$ & $0.41 \pm 0.14$ & 0.752 \\
\hline$\beta_{\text {ZEN14G }}\left(\mathrm{h}^{-1}\right)$ & $1.9 \pm 0.58$ & $<$ & $8.2 \pm 2.6$ & 0.006 \\
\hline 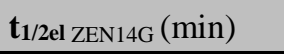 & $26 \pm 9.8$ & $>$ & $5.8 \pm 1.7$ & 0.025 \\
\hline $\mathrm{C}_{\mathbf{0}}(\mathrm{nmol} / \mathrm{mL})$ & $1.9 \pm 0.75$ & $=$ & $2.6 \pm 1.2$ & 0.414 \\
\hline Syst.Hydr. (\%) & $19 \pm 4.3$ & $=$ & $20 \pm 1.6$ & 0.748 \\
\hline FRAC (\%) & $94 \pm 7.8$ & $>$ & $61 \pm 15$ & 0.026 \\
\hline $\mathbf{C}_{\max }(\mathrm{nmol} / \mathrm{mL})$ & $0.22 \pm 0.10$ & $=$ & $0.58 \pm 0.25$ & 0.168 \\
\hline $\mathbf{t}_{\max }(\min )$ & $25 \pm 12$ & $=$ & $23 \pm 14$ & 0.902 \\
\hline
\end{tabular}

Values are presented as mean \pm standard deviation. Data of 8-week-old pigs are adopted from Catteuw et al. (Catteuw et al. 2019). CLZEN = total body clearance of ZEN; CLZEN14G = total body clearance of ZEN14G; QZEN14G = intercompartmental flow of ZEN14G; CLZEN14G $\rightarrow$ ZEN $=$ total body clearance of ZEN14G by systemic hydrolysis to $\mathrm{ZEN} ; \mathrm{Vd}=$ volume of distribution $\mathrm{Vc}=$ central volume of distribution; $\mathrm{Vp}=$ peripheral volume of distribution; $\mathrm{ke} / \beta=$ elimination/disposition rate constant; $\mathrm{t}_{1 / 2 \mathrm{el}}=$ elimination half-life; FRAC $=$ absorbed fraction of the administered dose in either form; $\mathrm{C}_{0}=$ plasma concentration of the administered mycotoxin at time of IV administration; $\mathrm{C}_{\max }=$ maximal plasma concentration; $\mathrm{t}_{\max }=$ time to maximal plasma concentration; Syst. Hydr. $=$ percentage of the administered dose of ZEN14G that is systemically hydrolysed to ZEN; $<,>,==$ significantly lower $(\mathrm{p}<0.05)$, significantly higher $(\mathrm{p}<0.05)$, not significantly different $(\mathrm{p} \geq 0.05)$, respectively, compared to results in 8 -week-old pigs. 
Supplementary Figure 1 Major metabolic pathways of zearalenone (ZEN) in humans and animals. CYP = cytochrome P450 enzymes; UGT = UDP-glucuronosyltransferase; HSD = hydroxysteroid dehydrogenase. GluO/OGlu = Oglucuronide. Adapted from EFSA 2011.<smiles>COC(C)CCCC(=O)CC/C=C/c1c(O)c(O)cc(O)c1C(=O)O</smiles>

13-hydroxy-zearalenone<smiles>COC(C)CCCC(=O)CC/C=C/c1cc(O)c(O)c(O)c1C(=O)O</smiles>

15-hydroxy-zearalenone<smiles>COC(C)CCCC(=O)[C@@H](O)CC/C=C/c1cc(O)cc(O)c1C(=O)O</smiles>

6/8-hydroxy-zearalenone<smiles>CC(C)C([Te])=[Te]</smiles><smiles>COC(=O)c1c(O)cc(O)cc1/C=C/CCCC(=O)CCCC(C)O</smiles>

3. UGT/<smiles>COc1cc(O)c(C(=O)OCCO)c(/C=C/CCCC(=O)CCCC(C)OC)c1</smiles>

Zearalenone-14-glucuronide<smiles>COc1cc(O)cc(/C=C/CCCC(=O)CCCC(C)OC)c1C(=O)O</smiles>

Zearalenone-16-glucuronide

Zearalenone 1. HSD<smiles>CC(CCC[C@H](O)CCC/C=C/c1cc(O)cc(O)c1C(=O)OC(C)(C)C)OC(=O)C(C)C</smiles>

3. UGT<smiles>CC(CCCC(O)O)OC(=O)c1c(O)cccc1/C=C/CCC[C@H](C)O</smiles><smiles>COC(=O)c1c(/C=C/CCC[C@H](O)CCCC(C)OC)cc(O)cc1OC</smiles>

$\alpha / \beta$-zearalenol-16-glucuronide 